\title{
Estado y antropología en México y Estados Unidos: reflexiones sobre los Proyectos Tarascos
}

\author{
Robert V. Kemper* \\ SOUTHERN METHODIST UNIVERSITY
}

La relación entre "el Estado y la antropología”, particularmente en cuanto a su relevancia para el desarrollo del trabajo antropológico en Michoacán, puede ser entendida mediante un análisis del Proyecto Tarasco, que efectivamente incluyó distintos episodios de trabajo de campo y distintos propósitos académicos y políticos. Estos proyectos -incluyendo los de Sáenz en Carapan, Swadesh en Paracho, Beals en Cherán y Foster en Tzintzuntzan- representaron una convergencia producto de los intereses de los gobiernos de México y Estados Unidos; una convergencia surgida, sin duda, de las circunstancias especiales de esa época, es decir, fines de la Gran Depresión y el periodo de la Segunda Guerra Mundial. Estos trabajos se realizaron gracias al sustancial apoyo económico y logístico proveído por numerosas agencias gubernamentales, tanto en México, como en Estados Unidos. En este sentido, los antropólogos que participaron en el Proyecto Tarasco no fueron simples "intermediarios intelectuales", sino "intermediarios institucionales" en su papel de agentes de sus respectivos gobiernos.

(Proyecto Tarasco, Carapan, Cherán, Tzintzuntzan, Michoacán)

* rkemper@mail.smu.edu Quisiera dar las gracias a Marco Calderón, director del Centro de Estudios Antropológicos de El Colegio de Michoacán, por la invitación a participar en la celebración del xxx aniversario del CEA. Además, quiero agradecer a todos mis amigos en el CEA, sobretodo a Andrew Roth-Seneff, por su cooperación durante todos los años de la existencia de este importante centro regional. También me es muy grato mencionar el excelente trabajo que hizo Paul Kersey en traducir al español la versión preliminar de la ponencia. También, agradezco la ayuda de la administración de los Archivos Antropológicos Nacionales en Washington, D.C., por su fina atención durante las dos estancias que hice para ver las 18 cajas que contienen los documentos del Instituto de Antropología Social de la Smithsonian Institution. 


\section{INTRODUCCIÓN}

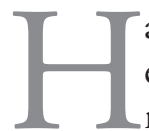
ace algunos años en su excelente tratado de "Nacionales y extranjeros en la historia de la antropología mexicana”, desarrollo de la antropología mexicana en estos términos: "Paradójicamente, la práctica antropológica mexicana ha implicado desde sus orígenes la presencia de extranjeros que a menudo han aportado financiamiento, pericia y liderazgo para empresas de investigación con o sin participación de estudiosos mexicanos-. Esto ha creado cierta tensión entre nacionales y extranjeros en el terreno profesional".

Esta paradoja fue especialmente evidente en la primera mitad del siglo xx, entre la fundación de la Escuela Internacional de Arqueología y Etnología Americana por Franz Boas, Eduardo Seler y George Engerrand en 1910, hasta las diversas actividades realizadas por el Instituto de Antropología Social de la Smithsonian Institution en México en el periodo 1943-1952. ${ }^{1}$ Los resultados de esos proyectos han dejado su huella en la antropología, no sólo en México sino también en Estados Unidos e, incluso, más allá.

Uno de los más influyentes proyectos de investigación en que participaron académicos extranjeros fue conocido como el Proyecto Tarasco. En realidad, no se trata de un solo proyecto, sino de una serie de actividades de índole lingüística y etnográfica con cierta interrelación que empezó hacia finales de la década de 1930 y continuó por unos diez años. Esas diversas iniciativas fueron emprendidas por un también diverso conjunto de antropólogos, lingüistas y otros académicos que representaron a, y fueron apoyados por, importantes agencias de los gobiernos de México y Estados Unidos. No obstante, cada uno llevó por nombre "El proyecto tarasco" y resaltó la colaboración que existía entre integrantes nacionales y extranjeros. Median-

${ }^{1}$ Recopilar una lista completa de los proyectos antropológicos conducidos entre 1910 y 1952 por académicos extranjeros con el patrocinio de instituciones extranjeras está más allá del alcance de esta presentación. Los interesados en entender el amplio rango de esos proyectos pueden consultar las historias estándares de la antropología mexicana sobre esa época (por ejemplo, Beals 1993; Hewitt de Alcántara 1984; Kemper 1993; Lameiras 1979; Rutsch 1996). 
te el presente examen detallado de estos Proyectos Tarascos espero iluminar el tema de este Congreso; ${ }^{2}$ es decir, la relación entre "el Estado y la antropología", particularmente en cuanto a su relevancia para el desarrollo del trabajo antropológico aquí en Michoacán.

En un sentido importante, los proyectos antropológicos realizados en las tierras altas de Michoacán en los treinta y cuarenta representaron una convergencia producto de los intereses de los gobiernos de México y Estados Unidos; una convergencia surgida, sin duda, de las circunstancias especiales de esa época. Las tensiones creadas entre las dos naciones por la Gran Depresión de la década de 1930 y exacerbadas con la nacionalización petrolera del 18 de marzo de 1938, fueron en buena medida dejadas de lado cuando la amenaza de la guerra con Alemania (y, más tarde, Japón) se convirtió en una realidad compartida.

Por esta razón, es importante mencionar este más amplio contexto de la antropología en México y Estados Unidos antes de enfocar nuestra mirada en los Proyectos Tarascos. En este repaso de las acciones de los gobiernos que influyeron en la disciplina de la antropología, veremos claramente que los proyectos de investigación en Michoacán formaron parte de una más amplia iniciativa que pretendía incorporar a antropólogos, lingüistas y otros científicos sociales al servicio del Estado.

\section{LA ANTROPOLOGÍA DURANTE EL RÉGIMEN CARDENISTA,} I934-I940

Como escribí en un tratado anterior (Kemper 1993, 34) sobre el desarrollo de la antropología durante el periodo cardenista:

El Estado, representado por individuos e instituciones concentradas en la ciudad de México, jugó un poderoso papel en la transformación de la institucionalidad científica y educativa de México, respondiendo a las necesidades de asistencia técnica para tratar con los problemas de desarrollo

2 "Antropología y Estado en México", XXX Aniversario del Centro de Estudios Antropológicos de El Colegio de Michoacán, Zamora, Michoacán, 28 y 29 de mayo de 2009. 
nacional durante la gran depresión y la Segunda Guerra Mundial. La antropología fue afectada de manera particular, gracias al interés personal de Cárdenas en los problemas del México rural y el patrimonio cultural, dentro del cual la herencia indígena fue considerada de especial importancia.

El compromiso de Cárdenas con los asuntos indígenas y con la antropología en sí quedó de manifiesto en muchas maneras en su sexenio como presidente del país (1934-1940). Durante su régimen se crearon numerosas instituciones gubernamentales, incluidos el Departamento de Asuntos Indígenas (1936); el Departamento de Antropología en la Escuela de Ciencias Biológicas del Instituto Politécnico Nacional (IPN, en 1937); y el Instituto Nacional de Antropología e Historia (1939). Además, en agosto de 1940, el Departamento de Antropología del IPN y el INAH firmaron el "Plan de cooperación para la enseñanza de la antropología en México”, que luego fue ampliado para incluir a la Sección de Antropología de la Facultad de Filosofía y Letras de la Universidad Nacional Autónoma de México (UNAM).

Al enviar con anticipación una invitación a los organizadores del Congreso Internacional de Americanistas que celebraría su XXVII Congreso en México, Cárdenas fungió como el presidente honorario del evento durante sus actividades realizadas en la ciudad de México del 5 al 15 de agosto de 1939. El año siguiente, a las 8:00 pm del día 14 de abril de 1940, para ser preciso, Cárdenas dio el discurso inaugural del Primer Congreso Indigenista Interamericano con sede en "El Teatro Caltzontzin" en Pátzcuaro, Michoacán. En un acto posterior, Cárdenas reconoció oficialmente el carácter "profesional” de la "carrera del antropólogo", mediante un decreto presidencial emitido el 21 de octubre de 1940, sólo nueve días antes de dejar el puesto y pasar la presidencia a su sucesor, Manuel Ávila Camacho.

\section{LA ANTROPOLOGÍA DURANTE LA ADMINISTRACIÓN De Ávila Camacho, I940-I 946}

El ajuste en las políticas del gobierno mexicano hacia una dirección distinta a la que había caracterizado al régimen cardenista tuvo im- 
plicaciones importantes para la antropología durante los años de la guerra. El cambio más significativo en la investigación y formación etnológicas fue el aumento de la participación de Estados Unidos en asuntos mexicanos. Se firmaron acuerdos que permitían a antropólogos norteamericanos venir a la Escuela Nacional de Antropología e Historia en México en calidad de profesores visitantes. Los norteamericanos fungieron como investigadores responsables de importantes proyectos de investigación de campo en que se involucraron asimismo estudiantes y profesionistas locales. Además, participaron en el creciente número de congresos, mesas redondas y seminarios de índole etnológica patrocinados por organizaciones tanto mexicanas como internacionales. Pero, durante la presidencia de Ávila Camacho el enfoque de las investigaciones etnológicas empezó a alejarse de los aspectos históricos e indígenas para incluir a las comunidades campesinas y poblaciones mestizas.

Como consecuencia de las políticas adoptadas por la nueva administración, el Departamento de Asuntos Indígenas sufrió importantes recortes y más tarde, en diciembre de 1946, se convirtió en una dependencia de la Secretaría de Educación Pública. Pero esta solución resultó insostenible y, por lo tanto, casi toda actividad relacionada con los indígenas fue transferida al recién fundado Instituto Nacional Indigenista (cuya creación, originalmente programada para 1940, tardó hasta 1948, después de la administración avilacamachista).

Mientras tanto, el Departamento de Antropología del IPN experimentaba una transformación más benéfica. En 1941, se iniciaron clases en el Museo Nacional, y en el ańo siguiente el Departamento fue transformado en la Escuela Nacional de Antropología bajo la autoridad presupuestaria del INAH, y ya no del IPN (Cámara Barbachano 1961; Jiménez Moreno 1948-1949). Bajo la hábil dirección de Daniel Rubín de la Borbolla, la Escuela pronto se expandió para abarcar el campo de la historia y -ahora como el ENAH- estaba en camino a convertirse en una institución central de investigación y formación en todos los campos de la antropología en México. En ese tiempo, organizaciones como la Fundación Rockefeller, el Viking Fund, Inc. (creado en 1941, luego cambió su nombre a la Fun- 
dación Wenner-Gren para la Investigación Antropológica), la Fundación Guggenheim y el Instituto de Antropología Social (Institute of Social Anthropology) de la Smithsonian Institution ofrecían becas a estudiantes mexicanos y latinoamericanos.

En 1941, el Departamento de Antropología contaba con 21 profesores y tenía como integrantes de su Junta de Consejo a Alfonso Caso, Pedro Sánchez, A.L. Kroeber y Paul Rivet. Además, Ralph Beals y Alfonso Villa Rojas aparecían como profesores honorarios. Para 1946, el personal había crecido hasta incluir, aunque sólo en nombre, a 12 antropólogos físicos, 11 arqueólogos, 16 historiadores, 13 lingüistas y 10 etnólogos. Había además 10 profesores invitados, la mayoría de ellos antropólogos norteamericanos. A simple vista, el ENAH parecía contar con mejor personal y un mejor presupuesto que cualquier programa de antropología en Estados Unidos o Europa durante los años de la guerra.

En 1942, por presiones de Alfonso Caso, la Institución Carnegie en Washington y la Fundación Rockefeller acordaron apoyar a Sol Tax (doctor de la Universidad de Chicago que estudió con Robert Redfield y había sido asignado a un proyecto de campo en Guatemala) para que fuera profesor visitante en la Escuela de Antropología. Tras una serie de discusiones complicadas e intensas, Tax finalmente partió hacia México. La explicación que envió a R.L. Boke de la Oficina del Coordinador de Asuntos Interamericanos (Office of the Coordinator of Inter-American Affairs) de la Fundación Rockefeller (que había solicitado sus servicios) refleja importantes conexiones entre los intereses de la política exterior norteamericana, las actividades de ciertas fundaciones privadas y la investigación y formación etnológicas:

Su información telefónica de que el Departamento del Estado no cree que sea aconsejable que abandone el proyecto mexicano fue en verdad el factor decisivo. El Sr. Stevens (aunque con recelo) amablemente me había liberado para poder trabajar en su oficina y estaba preparado -también con recelo-a decepcionar a nuestros amigos mexicanos. Pero por la opinión de los informantes del Sr. Clintock del Departamento de Estado me di cuenta que quizá sería un error serio, tanto del punto de vista de nuestras relaciones con 
México como del de las relaciones entre el Departamento de Estado y el proyecto en que iba a trabajar (Tax, carta a Boke, 28 mayo 1942).

El caso del involucramiento de Tax en el programa de docencia e investigación de la Escuela de Antropología muestra que la participación norteamericana en la antropología mexicana iba en aumento conforme continuaba la guerra. Ahora, pasamos a una consideración más detallada de las políticas de Estados Unidos hacia América Latina y México en los años de 1930 y 1940.

\section{LA ANTROPOLOGÍA DURANTE LAS ADMINISTRACIONES RoOSEVELT-TRUMAN, I932-I952}

Las administraciones progresistas de Roosevelt y Truman en Estados Unidos promulgaron, a partir de la década de 1930 y hasta los cuarenta, políticas similares. Por una parte, el antropólogo John Collier fungió como un activista director de Asuntos Indios (Commissioner of Indian Affairs) de 1933 a 1945, un dirigente entregado con diligencia a mejorar las condiciones de las poblaciones indígenas de Estados Unidos durante la Gran Depresión. Pero las políticas culturales e indigenistas norteamericanas no fueron limitadas por las fronteras nacionales. Tres ejemplos bastarán para evidenciar el creciente interés de Estados Unidos hacia México y América Latina.

\section{La Oficina de Asuntos Interamericanos}

Primero, en el más amplio nivel, muchas políticas fueron establecidas a través de la Oficina de Asuntos Interamericanos (Office of Inter-American Affairs, bajo la supervisión del Departamento de Estado; también conocida por dos nombres más largos: Office for Coordination of Commercial and Cultural Relations between the American Republics, y Office of the Coordinator of Inter-American Affairs). Dirigida por Nelson Rockefeller, esta oficina fue creada en 1940 y operó hasta 1946, después de terminada la Segunda Guerra Mundial. Como notan Gisela Cramer y Ursula Prutsch (2006, 785): 
Para mediados de 1940, los avances militares de la Alemania nazi habían desencadenado una profunda preocupación en Estados Unidos. Si bien, las noticias sobre los acontecimientos en Europa predominaban, varios eventos en las Américas intensificaron el sentido de alarma en el gobierno, la comunidad comercial y los medios de comunicación. Debido a los trastornos que sufrían el comercio internacional y los flujos de capitales por la guerra, muchos países latinoamericanos parecían estar al borde de una severa caída económica. Se temía que semejantes problemas económicos podrían generar una desestabilización política que luego propiciaría la intervención de los países del Eje y las actividades de la "Quinta Columna". En pocas palabras, para muchos observadores en Estados Unidos, las repúblicas latinoamericanas parecían propensas a la penetración política, cultural, económica e, incluso, militar de la Alemania nazi y sus aliados.

En diciembre de 1940, antes de que Estados Unidos entrara en la guerra, un grupo de antropólogos norteamericanos interesados en América Latina organizaron una conferencia que dio a luz un comité en el Consejo Nacional de Investigación (National Research Council, NRC). Más tarde, en marzo de 1942, el NRC, el Consejo Americano de Sociedades Académicas (American Council of Learned Societies) y el Consejo de Investigación en las Ciencias Sociales (Social Science Research Council, sSRC) crearon un Comité Conjunto para coordinar sus intereses en América Latina. En ese mismo mes, la Smithsonian Institution formó un Comité de Guerra y luego, en junio de ese año, prestó oficinas a la recién creada Junta Etnográfica (Ethnographic Board), encargada de "hacer fácilmente accesible a agencias militares y de la guerra en Washington [...] información regional específica y datos evaluados de personal" (Bennett 1947, v). Esas actividades fueron precisamente las que la Oficina del Coordinador de Asuntos Interamericanos debía agilizar mediante su nuevo Instituto de Asuntos Interamericanos.

\section{El Instituto de Antropología Social}

Como George M. Foster (1979, 205-206) informó en su historia de este Instituto: 
El Instituto de Antropología Social [ISA] fue el intento por parte de Steward de ayudar a satisfacer necesidades que él previó. Se basó el Instituto en un plan tripartito: 1) crear programas cooperativos de enseñanza e investigación en antropología y las ciencias sociales afines en instituciones de nivel universitario en países latinoamericanos; 2) realizar investigación intensiva en comunidades rurales contemporáneas del tipo que pudieran experimentar una rápida modernización; y, 3) publicar informes de investigación en una nueva serie de la Smithsonian Institution, misma que estaría disponible a los planeadores de los varios países involucrados y proporcionaría los datos socioculturales requeridos [...] El Instituto fue sólo uno de los muchos proyectos creados por el gobierno norteamericano a través de la llamada "Política del Buen Vecino" (Good Neighbor Policy) para América Latina, un conjunto de actividades que tomó forma a mediados de los treinta, todas ellas diseñadas para estimular lazos culturales y de amistad más cercanos con países en América Latina. Con la entrada de Estados Unidos en la Segunda Guerra Mundial, el impulso de esta política se aceleró, y había dinero para varios proyectos que, en tiempos de paz, probablemente no habrían recibido apoyo.

\section{Un breve recuento del Proyecto tarasco: de Carapan a Tzintzuntzan}

Esta convergencia de los intereses de los gobiernos de México y Estados Unidos no explica por sí sola la elección de las tierras altas de Michoacán como el escenario de varias iniciativas, cada una de ellas etiquetada en el Proyecto Tarasco. ¿Por qué fue escogida la región tarasca, y no la de los zapotecos de Oaxaca, o la de algún otro grupo indígena? La razón me parece bien clara: esta región de México, cuyos pueblos rurales habían sufrido levantamientos religiosos y agrarios desde los veinte, fue elegida porque era la tierra natal de la entonces más poderosa figura política de México; el presidente del país, general Lázaro Cárdenas.

A lo largo de su carrera, Cárdenas había manifestado un claro perfil proagrarismo, anticlerical y antifascista, así como un firme compromiso con el mejoramiento de la vida de los pueblos indígenas (Ginzberg 1999). Para citar sólo un, muy conocido, ejemplo, 
podemos mencionar lo ocurrido en los treinta en la antigua capital tarasca de Tzintzuntzan. Como observó George M. Foster (2000a, 49) en su famoso estudio de esta comunidad:

El general Cárdenas estaba imbuido de un deseo sentimental y humanitario de restaurar, aunque fuera de pequeña manera, la anterior grandeza de la capital tarasca y de aumentar el bienestar de la moderna población. Así, no fue por casualidad que el viejo kiosco apareciera en Tzintzuntzan, ni que las bancas recobraran la grandeza anterior. Asimismo, la carretera, la escuela, el sistema de agua, la electricidad, el teatro abierto al aire libre y la categoría política de municipio, todo se debe al [general Cárdenas].

\section{Carapan: Moisés Sáenz y la "Estación experimental DE LA INCORPORACIÓN DEL INDIO”}

Empezamos con lo que podría considerarse el Preproyecto Tarasco: el experimento educativo bilingüe que arrancó Moisés Sáenz a inicios de los treinta en el pueblo de Carapan, ubicado en el municipio de Chilchota y en la región conocida como La Cañada de los Once Pueblos. Este proyecto fue innovador por su esfuerzo por desarrollar un programa educativo bilingüe para la gente de habla tarasca en que se emplearon métodos para la enseñanza directa del espańol, sobre todo a través de técnicas auditivas que no implicaban la lectoescritura. Sáenz pudo contemplar ese radical experimento porque ocupó una serie de altos puestos directivos en la Secretaría de Educación. ${ }^{3}$ Desgraciadamente, el "experimento" en la estación de Carapan sólo duró seis meses y estaba aún incompleto cuando Sáenz se fue de Michoacán. No obstante, causó muy fuerte impresión en el entonces gobernador del estado, el general Lázaro Cárdenas, quien fue el primer alto funcionario en visitar la estación.

Respecto del proyecto de Sáenz hay varios puntos que ameritan mención. Primero, él había ido al campo mexicano antes para exami-

${ }^{3}$ En un momento, Sáenz ocupó un puesto en la Secretaría antes ocupado por Manuel Gamio, quien tuvo que dejarlo por presiones y quien, a finales de los veinte, fue a Estados Unidos a estudiar migrantes mexicanos con el apoyo profesional e institucional de Robert Redfield en la Universidad de Chicago y el apoyo financiero de la Social Science Research Council. 
nar varios programas de la Secretaría de Educación que usaban el español como medio de enseñanza entre los pueblos indígenas, y juzgó que eran fracasos. Esas experiencias comparativas lo llevaron a desarrollar un programa experimental para la meseta tarasca de $\mathrm{Mi}$ choacán y él mismo decidió participar directamente en esta iniciativa "misionera" en el campo de la lingüística aplicada y la educación. Desgraciadamente, no pudo sostener su participación directa más allá de seis meses y, poco después de su salida, el proyecto fue abandonado. Segundo, no cabe duda que este "preproyecto" fue estimulado por el contacto de Sáenz (miembro activo de la Iglesia Presbiteriana Nacional en México) con William Cameron Townsend, un misionero presbiteriano norteamericano con una enorme pasión por la educación de los pueblos indígenas (Gamio y Noriega 1961). Townsend, por su parte, desarrolló gran admiración por Cárdenas, cuya poderosa posición en la política mexicana permitió a su organización -fundada en 1936 bajo el nombre de Wycliffe Bible Translators que luego fue cambiado por el del Instituto Lingüístico de Verano (ILV) obtener el permiso del gobierno federal para llevar a cabo sus labores en México durante unas cuatro décadas, hasta que fue rescindido en 1979 (Colegio de Etnólogos y Antropólogos Sociales 1979). Tercero, como miembros activos de la iglesia presbiteriana ambos, Sáenz y Townsend, valoraban el compromiso de su Iglesia con proveer de servicios educativos y atención médica a los pueblos nativos.

\section{El primer Proyecto Tarasco: LINGÜistas EN LAS TIERRAS ALTAS DE MichoaCÁN}

Ese Preproyecto Carapan de Sáenz estimuló un programa más amplio de educación bilingüe para las tierras altas de Michoacán. Iniciado con el apoyo del Departamento de Asuntos Indígenas durante la administración cardenista, el primer Proyecto Tarasco implicó la participación de lingüistas con formación antropológica (algunos afiliados con iglesias protestantes) en la elaboración de un sistema fonético para el lenguaje tarasco y, posteriormente, su implementación en programas de educación bilingüe. Este proyecto ha sido descrito en una versión de primera mano por Ignacio M. del Castillo (1945, 146-148): 
La dirección del Proyecto Tarasco quedó encomendada el Dr. Morris Swadesh, uno de los más distinguidos filólogos norteamericanos, profesor de Lingüística de la Universidad de Wisconsin y destacado alumno de Edward Sapir, con extensa experiencia en el estudio de lenguas indígenas. El personal técnico estuvo integrado por el profesor Alfredo Barrera Vásquez, exdirector del Museo Arqueológico de Yucatán, renombrado mayista y destacado filólogo; por el Profr. Maxwell D. Lathrop, del Summer Institute of Linguistics, quien entonces llevaba ya dos ańos de estancia en la región lacustre de Michoacán y que habla muy bien el tarasco; por el Profr. Juan Luna Cárdenas, nahutlahto $[s i c]$ y presidente de la Sociedad Mexicana Hueytlatepecpanaliztli; por el Sr. Adrián F. León, entonces estudiante de lingüística en la Escuela Nacional de Antropología y actualmente filólogo del Museo Nacional; por la Srita. Frances León, estudiante de lingüística de la Universidad de Yale; y por el autor de estas líneas, comisionado en el "Proyecto Tarasco" por el Instituto Nacional de Antropología e Historia, en su calidad de filólogo del propio Instituto.

En el mes de julio de 1939 se instalaron las oficinas del Proyecto Tarasco en la Escuela Vocacional de Agricultura, de la población de Paracho, Michoacán, y los primeros pasos que se dieron consistieron en la preparación de maestros nativos. Con tal fin, el Dr. Swadesh, autorizado por el Departamento de Asuntos Indígenas, reunió un grupo de veinte estudiantes, entre hombres y mujeres, todos ellos purépechas genuinos y que por tanto hablaban corrientemente el tarasco [... .]

Una vez preparados así esos veinte jóvenes, quedaron capacitados para servir de maestros en las misiones alfabetizadoras, las cuales estaban compuestas por un técnico lingüista, como jefe, y por algunos de los maestros nativos. Poco a poco se fueron instalando misiones en los pueblos de los alrededores de Paracho, y personalmente tuvimos la oportunidad de encabezar la que trabajó en el pueblo de Arantepácua, a unos 18 o 20 kilómetros de Paracho, a través de la sierra [...]

Además, según del Castillo $(1945,148)$ :

En todos [los alumnos], nińos y adultos, se despertó un deseo vehemente de aprender, como que por primera vez comprendían al maestro, ya que recibían la instrucción en su propio idioma. Estaban asombrados, y así me 
lo hicieron saber varias veces, de que su lengua, el idioma tarasco, pudiera escribirse. Para ellos sólo era concebible que el español podía ser escrito y leído, pero nunca el tarasco. De manera que se les presentaba un mundo nuevo, lleno de bellas perspectivas, al ver que podían trasladar fielmente a un papel sus pensamientos y sus ideas, y que sus medios de comunicación se ampliaban. ¡Sólo entonces pudimos darnos cuenta de lo que para el indígena significa instruirse en su propio idioma!

\section{LA PRIMERA ASAMBLEA DE FILÓLOGOS Y LINGÜISTAS}

La expansión de este exitoso programa de alfabetismo indígena hacia otras regiones requería el desarrollo de herramientas lingüísticas estandarizadas, necesidad que condujo a la organización de la Primera Asamblea de Filólogos y Lingüistas, celebrada en la ciudad de México del 9 al 17 de mayo de 1939. Con el patrocinio del Departamento de Asuntos Indígenas y el Instituto Politécnico Nacional, el Departamento de Antropología de la Escuela Nacional de Ciencias Biológicas fue el responsable de la Asamblea. La importancia de las instituciones que apoyaron el evento se refleja en la primera cláusula del reporte publicado dedicada a enumerar las organizaciones allí presentes. La lista incluye al: "Departamento de Asuntos Indígenas, Departamento de Antropología, Departamento Agrario, Group for American Indian Languages, Instituto Nacional de Antropología e Historia, Academia de la Lengua Maya de la Península de Yucatán, Summer Institute of Linguistics, Instituto Panamericano de Geografía e Historia, Sociedad Huey Tlatekpanalitztle, Sociedad Mexicana de Antropología, y Linguistic Society of America”.

Esta importante reunión contó con la asistencia de 45 de las 50 personas que fueron invitadas a contribuir a resolver los complejos problemas de la ortografía lingüística surgidos a partir de la experiencia del Proyecto Tarasco. La "Lista de Congresistas" incluye los nombres de distinguidos académicos y oficiales de gobierno, tales como Alfredo Barrera Vásquez, Daniel Rubín de la Borbolla, Sherburne F. Cook, Alfonso Caso, Ignacio M. del Castillo, Luis Chávez Orozco, Andrés Henestrosa, Wigberto Jiménez Moreno, Paul Kirchhoff, Maxwell C. Lathrop y Sra., Adrián F. León, Juan Luna Cárdenas, Norman A. 
ALFABETO dEL CONSEJO dE LENGUAS INDIGENAS

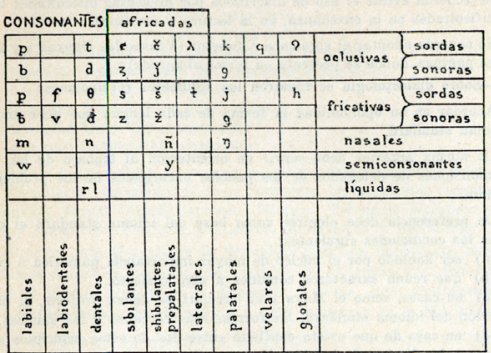

VOCALES

anteriores posteriores

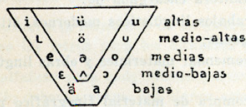

Variaciones opcionales. $\ell=t s ; z=d z ; \breve{c}=\varsigma, c h ; \breve{J}=d z$; $\breve{s}=s, x$; $\check{z}=\xi ; \lambda=\star, z, t l ; \lambda=d l$ cuando hay tonto la sonora comela sorda; $\eta=n g ; ?=$ ?.

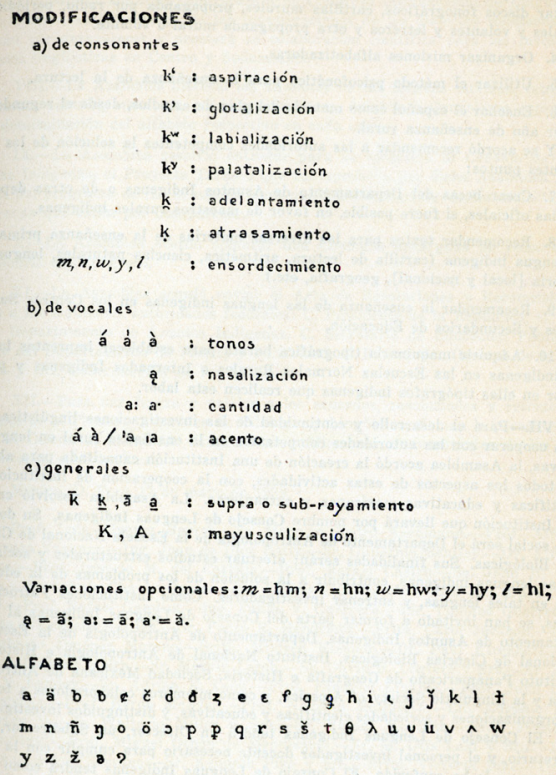

Figura 1. Morris Swadesh, Wigberto Jiménez Moreno, Juan Luna Cárdenas, Norman McQuown, Jorge Vivó y Daniel Rubín de la Borbolla, Memoria de la Primera Asamblea de Filólogos y Lingüistas, México, 1939.

McQuown, Miguel Othón de Mendizábal, Kenneth Pike y Sra., Jacques Soustelle, Morris Swadesh, William C. Townsend y Sra., Alfonso Villa Rojas, Jorge A. Vivó Escoto y Roberto J. Weitlaner. Además, se extendieron invitaciones a la Asamblea a representantes de las siguientes lenguas indígenas: náhuatl, maya, otomí, zapoteco, mixteco, totonaco, mazateco, tarasco, huaxteco, chinanteco, popoluca, cuitlateco, matlatzinca y cuicateco.

Uno de los principales resultados de la Asamblea fue la creación de un "Consejo de Lenguas Indígenas", cuya más importante contribución consistió en adoptar un alfabeto estándar para las lenguas indígenas (véase figura 1). Cabe señalar que las personas que asistieron a la Asamblea acordaron que los estatutos aprobados para el nuevo Consejo de Lenguas Indígenas serían entregados personalmente por William C. Townsend al C. Presidente de la República, 
General Lázaro Cárdenas, y al C. Jefe del Departamento de Asuntos Indígenas, el Profr. Luis Chávez Orozco (Swadesh et al. 1939).

Desgraciadamente, el éxito obtenido durante la Asamblea con la creación de una ortografía estándar para las lenguas mesoamericanas no bastó para sostener el Proyecto Tarasco más allá del sexenio de Cárdenas, y es que para la administración de Manuel Ávila Camacho los programas "socialistas", incluidos los enfocados en el alfabetismo y la educación indígena, resultaron mucho menos atractivos que durante el cardenismo. En las palabras de Ignacio M. del Castillo $(1945,150)$ :

Los impugnadores del Proyecto Tarasco creyeron encontrar semejanzas entre los sistemas usados en este experimento y los sistemas de alfabetización usados por la U.R.S.S. (a pesar de que los métodos que ésta empleara, fueron estrictamente científicos) y eso fue motivo para cortar la continuidad de aquellos trabajos, haciéndolos morir cuando empezaban a dar sus primeros frutos y no dejándolos alcanzar su completa madurez. Sin embargo, la experiencia obtenida entonces no ha muerto.

El segundo Proyecto Tarasco: Beals (ucla) y Rubín de la Borbolla (IPN) en Cherán

Al mismo tiempo que el primer Proyecto Tarasco estaba desarrollándose en la región de Paracho, una segunda iniciativa surgía de las fecundas mentes de dos antropólogos: Ralph L. Beals, profesor del Departamento de Sociología y Antropología de la Universidad de California en Los Ángeles (UCLA), y Daniel Rubín de la Borbolla, Coordinador del recién creado Departamento de Antropología de la Escuela de Ciencias Biológicas del Instituto Politécnico Nacional en la ciudad de México.

Su reporte sobre esta "iniciativa cooperativa" fue presentado conjuntamente como una contribución oficial de parte de la Delegación Mexicana al Primer Congreso Indigenista Interamericano en su reunión en Pátzcuaro, Michoacán, del 14 al 24 de abril de 1940. Sin duda, el principal fruto de ese Congreso fue el acuerdo al que llegaron los representantes de 18 naciones para crear el Instituto 
Indigenista Interamericano, organismo cuyo financiamiento provendría de contribuciones de todos los países miembros, y cuya sede sería la ciudad de México. De las 52 recomendaciones aprobadas por los congresistas, la octava trató de los "Planes Integrales en la Investigación de los Pueblos Indígenas” y declaró en la página 14 del Acta Final: "Que el Instituto Indigenista Interamericano fomente estudios integrados y coordinados de investigaciones similares a los planeados en la ZONA TARASCA, por el Instituto Politécnico Nacional de México y la Universidad de California (proyecto tarasco) y que estimule, especialmente, proyectos de cooperación interamericana de este carácter. (Aprobada abril 23)".

El informe de Borbolla y Beals fue publicado poco después del Congreso en la revista American Anthropologist (1940, 708-712). Inician su informe lamentando la escasez de estudios etnográficos contemporáneos sobre una región cultural de tanta importancia histórica; luego comentan que el acceso a la región se había agilizado poco antes con la inauguración de una carretera que comunicó a la ciudad de México con Guadalajara, y que tenía una rama que llevaba a Uruapan. Declararon que $(1940,708)$ :

Un grupo numeroso, relativamente homogéneo y concentrado está, entonces, disponible para su estudio en condiciones de cambio; presenta un excelente campo para la antropología social. Es altamente probable que cualquier estudio conducido a conciencia tenga un alto grado de utilidad práctica para todo administrador progresista deseoso de amortiguar el choque del cambio cultural y de canalizar este cambio por direcciones constructivas.

En el informe, los autores describieron en detalle los orígenes del proyecto:

La idea de un estudio minucioso e integrado de la Tarasca [sic] tuvo su incepción en el Departamento de Antropología de la Escuela Nacional de Ciencias Biológicas del Instituto Politécnico Nacional en la Ciudad de México. Algunas ideas preliminares fueron discutidas por el Dr. Paul Kirchhoff del Instituto Politécnico Nacional y el Dr. Ralph Beals en diciem- 
bre de 1938. En la primavera de 1939, el Dr. Daniel Rubín de la Borbolla, coordinador del Departamento de Antropología del Instituto Politécnico Nacional, visitó California y habló a más detalle del proyecto con el Dr. Beals y el Dr. A.L. Kroeber y, en ese momento, hizo algunas sugerencias tentativas sobre la cooperación entre la Universidad de California, el Instituto Politécnico Nacional y la Oficina de Asuntos Indígenas de México. Como resultado de esta visita, en el verano de 1939, el Dr. Beals hizo un corto viaje de sondeo patrocinado por estas tres instituciones. En ese momento, se elaboró también un plan de investigación preliminar, mismo que fue entregado más tarde a las instituciones. Como consecuencia de estas actividades, las tres instituciones han aceptado la idea de un estudio cooperativo, y en 1940 el trabajo de campo cooperativo inició en lo que se espera sea una extensa colaboración entre la Oficina de Asuntos Indígenas, la Universidad de California y el Instituto Politécnico Nacional [...] [E]l programa de investigación debe mucho al trabajo del Dr. Manuel Gamio en Teotihuacán, a la labor del Dr. Robert Redfield, al programa de investigación del Instituto Carnegie de Washington y al trabajo, aún inédito, del Dr. Miguel O. Mendizabal sobre el valle de Mezquital (1940, 709).

Enseguida de una extensa descripción de las perspectivas de éxito de un complejo estudio de campo a largo plazo que anticipaba las contribuciones de numerosos célebres etnólogos, arqueólogos, antropólogos físicos y lingüistas, los autores reconocieron que "pudiera parecer demasiado ambicioso" $(1940,711)$. Hubo la esperanza de que otras instituciones, aparte de la Universidad de California y el IPN, contribuyeran con personal y fondos a esta iniciativa. Desafortunadamente, en realidad este proyecto nunca alcanzó las dimensiones pretendidas, en parte por la situación de Beals. Después de pasar el año académico de 1941-1942 en Michoacán, Beals tuvo que regresar a su labor docente en uCla en el año académico 1941-1942. A petición de Julian Steward, su antiguo amigo de Berkeley, alcanzó a salir de California para trabajar en Washington, D.C. en $1942-$ 1943. Este círculo de conexiones con la Universidad de California se completó cuando Beals arregló que George M. Foster, recién graduado del programa de antropología de la Universidad de California, lo reemplazara en UCLA durante el año académico de 1942-1943. 
Allá en Washington, Beals fue contratado por la Smithsonian Institution al puesto de "Coordinador de Estudios Étnicos", que implicaba tomar la responsabilidad de organizar la recién formada Sociedad Interamericana de Antropología y Geografía (Inter-American Society of Anthropology y Geography) y de redactar su nueva revista, Acta Americana, trabajo que conservó durante la breve existencia de esa publicación (1943-1948). ${ }^{4}$ Por orden del presidente de la Universidad de California Beals tuvo que regresar de nuevo a Los Ángeles para dar clases durante el año académico 1943-1944 y los años siguientes.

Las publicaciones logradas en el contexto de este Proyecto Tarasco incluían informes sobre el trabajo de campo realizado en, y alrededor de, el pueblo de Cherán en la meseta tarasca. A dos breves artículos - "The Diet of a Tarascan Village" ("La dieta de un pueblo tarasco", de Beals y Hatcher, 1943), y "Games of the Mountain Tarascans" ("Juegos de los tarascos de la montaña”, de Beals y Carrasco 1944)- siguieron dos monografías, Houses and House Use of the Sierra Tarascans (Vivienda y el uso de vivienda entre los tarascos de la sierra, Beals, Carrasco y McCorkle 1944), y Cherán: A Sierra Tarascan Village (Cherán: Un pueblo tarasco de la sierra, Beals 1946). A pesar del compromiso de los coordinadores de publicar los principales resultados de las investigaciones del Proyecto Tarasco en México y Estados Unidos, la monografía Cherán es la única que hasta ahora ha sido traducida al español (Beals 1992), esto gracias a El Colegio de Michoacán.

Estas publicaciones reflejaron las relaciones sociales y académicas entre Beals, Steward y Foster, cada uno de los cuales tuvo un papel en el Instituto de Antropología Social durante y después de los años de la guerra. En realidad, Beals logró publicar sus monografías como los primeros dos números de una serie emitida por dicho instituto debido a los constreńimientos presupuestarios impuestos por la

${ }^{4}$ Quizá las dos más conocidas contribuciones que aparecieron en esa revista fueron el artículo de Paul Kirchhoff titulado "Mesoamérica" (1943, 92-107) y el reporte del Comité de Antropología Latinoamericana (Committee on Latin American Anthropology) del National Research Council titulado Research Needs in the Field of Modern Latin American Culture (Necesidades de investigación en el campo de la cultura moderna latinoamericana) $(1948,17-25)$. 
apropiación anual del Congreso norteamericano. Cada año, Steward tenía que mostrar cómo se había gastado los fondos del año anterior y, además, justificar el presupuesto solicitado para el año venidero. Como director, había programado ciertas publicaciones para el primer año presupuestal del ISA, que corría del 1 de julio de 1943 a finales de junio de 1994; pero, como ninguno de los proyectos de campo recién iniciados bajo el patrocinio del ISA había llegado al punto en que pudiera generar resultados publicables, la solución que Steward encontró consistió en autorizar la edición de los resultados del trabajo de campo del proyecto relacionado en tierras tarascas que su amigo Beals estaba a punto de terminar. Al principio de 1944, Steward escribió a su jefe, C. G. Abbot, que el presupuesto incluyera una cantidad para publicar "una serie de trabajos derivados de estudios ya cumplidos entre los tarascos de México" (carta de Steward a Abbot, fechada 5 de enero de 1944, ISA Papers, National Anthropological Archives, Washington, D.C.).

\section{El último Proyecto Tarasco: el isa-Foster y Tzintzuntzan}

A mediados de 1943, Julian Steward estaba tan seguro que el Congreso de Estados Unidos aprobaría los fondos para establecer el Instituto de Antropología Social que escribió una carta a su jefe, C. G. Abbot, Secretario de la Smithsonian Institution. En este comunicado, Steward describió con claridad su plan para implementar una serie de Institutos en América Latina, sobretodo en México:

Otro factor que favorece establecer el programa en México es que el Dr. Beals debe estar disponible durante el año próximo. Con muy buena reputación en México, a través de sus años de investigación y por sus lazos con científicos mexicanos y su experiencia colaboradora en el proyecto tarasco, él es la persona ideal para encargarse del Instituto durante su primer año experimental. Estos factores favorables deben asegurar resultados impresionantes antes del fin del primer año en México [. . .] Recomiendo entonces que México sea seleccionado como el primer país en que se establezca el Instituto de Antropología Social y que la Smithsonian Institution 
pida a la Universidad de California que se otorgue un sabático al Dr. Beals para el año académico próximo. Así el Dr. Beals tendrá la oportunidad de desarrollar el programa mexicano durante un ańo después de que regrese a la universidad y el proyecto quedará dirigido por otro antropólogo (carta de Julian Steward a C. G. Abbot, fechada 13 de mayo de 1943, Isa Papers, National Anthropological Archives, Washington, D.C.).

En otro comunicado con su jefe, Steward explicó en mayor detalle el programa que quisiera llevar a cabo en México:

En cooperación con el Instituto Nacional de Antropología e Historia, el equipo del Instituto dará adiestramiento a los alumnos de la Escuela Nacional de Antropología y llevará a cabo un programa de investigación amplio y de largo plazo entre los indios tarascos, un área y proyecto en que el ex-presidente Cárdenas ha tenido intereses muy profundos (carta de Julian Steward a C. G. Abbot, fechada 5 de enero de 1944, pp. 2-3, ISA Papers, National Anthropological Archives, Washington, D.C.).

Desafortunadamente, a mediados de 1943, Beals dejó Washington para regresar a su puesto de profesor en UCLA. A la vez, Foster salió de UCla (donde había rentado la casa de Beals durante el año que fue su suplente) para dirigirse a Washington, donde obtuvo un puesto como "analista en ciencias sociales" en el Instituto de Asuntos Interamericanos, dependencia de la Oficina del Coordinador de Asuntos Interamericanos, dirigida por Rockefeller. Allí, escribió unos informes de fondo para el personal del gobierno norteamericano que viajaba a diferentes áreas de América Latina. Foster y sus colegas aprovecharon fuentes de fácil acceso para producir reportes que los burócratas luego sellarían como "Clasificados". Al reflexionar años después sobre esta experiencia, Foster dijo que el mantener en secreto estos reportes no tenía ningún sentido y que, en realidad, era contraproducente para la realización de labores efectivas (Foster 2000b, 119).

Tras unos cuatro meses en Washington, Foster fue abordado por Steward sobre la posibilidad de cambiarse al nuevo Instituto de Antropología Social e ir a México -otra vez como suplente de Beals- 
como Director de Programa en el primer intento del ISA de hacer realidad su misión de enseñar y hacer investigación en colaboración con académicos y estudiantes locales. Según Foster,

Julian me preguntó si me gustaría ir a México. Me dijo que de hacerlo tendría que trabajar entre los tarascos, lo que me entusiasmó porque ya había estado en el área como turista en 1940, así que acepté. Pero dije, "No te lo puedo prometer porque estoy sujeto al servicio militar (draft)". Sólo estuve con el Instituto de Asuntos Interamericanos unos cuatro meses. En septiembre fui al Smithsonian con la esperanza de irme pronto a México. Pero la firma del convenio entre nuestro gobierno y el de México tardó meses y meses.

El contenido de este convenio quedó desglosado en un documento titulado "Intercambio de apuntes que constituyen un acuerdo relacionado con un programa cooperativo de trabajo e investigación antropológica en México. México, 4 de diciembre de 1943 y 19 de abril de 1944" (Exchange of notes constituting an agreement relating to a co-operative program for anthropological research and investigation in Mexico. Mexico, 4 December 1943 and 19 April 1944) que firmaron Ezequiel Padilla (secretario de Relaciones Exteriores de México) y George S. Messersmith (embajador de Estados Unidos en México) en representación de sus respectivos gobiernos. Aparte de proporcionar una extensa información sobre las cualidades de Foster para desempeñar el encargo propuesto en representación de la Smithsonian Institution, este documento también esboza los objetivos generales acordados por la Smithsonian y el ENAH del INAH:

1. Cooperar en la formación del personal de investigación de Estados Unidos y México en las técnicas de investigación en las ciencias sociales, la lingüística y la antropología física;

2. Cooperar en un programa de investigación de campo a largo plazo entre los tarascos y otros indios mexicanos que aliente la formación de estudiantes en el trabajo de campo y proporcione datos básicos para un entendimiento de los pueblos nativos de las Américas; programa que será integrado en el plan ya elaborado y realizado parcialmente 
por el Instituto Nacional de Antropología e Historia y la Universidad de California; y

3. Publicar los resultados de la investigación bajo los auspicios y en las formas e idiomas que, en opinión de la Smithsonian Institution y el Instituto Nacional de Antropología e Historia, resulten más adecuados.

Para este fin, el gobierno de Estados Unidos, a través de la Smithsonian Institution, acuerda:

(a) proporcionar los servicios de un antropólogo social, un geógrafo cultural y un lingüista o etnólogo;

(b) pagar los gastos de sus empleados mientras participan en la investigación de campo; $y$

(c) financiar la publicación de una parte de la investigación cooperativa.

El gobierno de los Estados Unidos Mexicanos, a través del Instituto Nacional de Antropología e Historia de la Secretaría de Educación Pública, acuerda:

(a) proporcionar los servicios de la facultad de la Escuela Nacional de Antropología y sus agencias colaboradoras, espacio para oficinas, laboratorios, libros e instalaciones docentes, y estudiantes para la formación universitaria y la capacitación en la investigación de campo;

(b) pagar los gastos de su personal mientras participa en la investigación de campo; $y$

(c) financiar la publicación de una parte de los resultados de la investigación.

Este convenio revela claramente el por qué Steward le había dicho a Foster que trabajaría en la región tarasca. Esta nueva iniciativa de la Smithsonian, mediante el IsA, quedó definida como una extensión de aquel Proyecto Tarasco que Beals y Rubín de la Borbolla habían llevado a cabo en 1940-1941. Dada la afiliación de Beals con el ISA y el papel de Rubín de la Borbolla como director del Departamento de Antropología del IPN y, más tarde, de la Escuela Nacional de Antropología (del INAH), esta conexión debió haber parecido lógica a Steward. Además, le brindó la justificación que necesitaba para proceder a editar las dos monografías sobre Cherán escritas por Beals y sus colegas como las Publicaciones núm. 1 y núm. 2 del ISA.

Al mismo tiempo que Estados Unidos y México empezaban a negociar su acuerdo cooperativo, el secretario de la Smithsonian 
Institution intentaba evitar que Foster fuera llamado al servicio militar. Fue por esta incertidumbre sobre su estatus respecto del servicio que la Smithsonian se esforzó tanto para que quedara exento. En un último y desesperado intento de proteger a Foster de su obligación militar y así poder contar con sus servicios para el proyecto del IsA en México, el Dr. C. G. Abbot (secretario de la Smithsonian) envió una carta a la Srita. Antoine Stallone, encargada del Servicio Selectivo de la Junta Local núm. 67 en Berkeley, California, donde fue registrado Foster. La carta del secretario, fechada el 18 de diciembre de 1943 (ISA Papers, National Anthropological Archives, Washington, D.C.), es reveladora respecto de los propósitos políticos atrás del programa cooperativo del ISA:

Estimada Srita. Stallone:

La Smithsonian Institution ha contratado al Dr. George M. Foster, Jr. (Orden núm. 734, número de Serie 1884) para trabajar en México en el desarrollo de un programa en uno de los proyectos del Comité Inter-departamental para la Cooperación con las Repúblicas Americanas (Interdepartmental Committee for Cooperation with the American Republics) patrocinado por el Departamento del Estado y realizado por la Smithsonian Institution. Financiado por una apropiación especial del Congreso, pretende fortalecer los lazos entre las Repúblicas Americanas a fin de contrarrestar la influencia de la idealogía [sic] del Eje que ha penetrado en estos países y conducir investigación en el marco de muchos importantes proyectos relacionados con el esfuerzo de guerra.

El trabajo de la Smithsonian en México es una colaboración con el Instituto Nacional de Antropología e Historia. Incluye la formación universitaria para promover el pensamiento norteamericano en las ciencias sociales en las universidades de México y la investigación en las ciencias sociales entre los pueblos nativos de México; este último diseñado para lograr un entendimiento más pleno de los pueblos y proveer información necesaria para los muchos programas de acción de otras agencias del gobierno de Estados Unidos que actualmente están en pie.

El gobierno de Estados Unidos está tan profundamente comprometido con este programa que su suspensión en este momento tendría un efecto extremadamente negativo en las relaciones inter-Americanas y, ade- 
más, impediría el desarrollo de aspectos importantes del proyecto. Dado que el Dr. Foster está a cargo de este trabajo y desempeña las tareas más esenciales sería imposible llevarlo a cabo si él no estuviera disponible. El Dr. Foster es irremplazable en este puesto porque combina el conocimiento de la antropología social, la habilidad de enseñar en español y antecedentes de experiencia en México que hoy son casi imposibles de duplicar.

En respuesta a una petición del 18 de agosto de 1943, el Dr. Foster recibió el permiso de ausentarse de los Estados Unidos para llevar a cabo este trabajo en México hasta el 22 de febrero de 1944, fecha en la cual se solicitaría una renovación.

Solicitamos respetuosamente que el Dr. Foster sea clasificado de tal manera que pueda realizar el trabajo de la Smithsonian Institution en México, y que se extienda su permiso de ausentarse de los Estados Unidos. Atentamente,

[C. G. Abbot]

\section{Secretario}

Pero la carta ${ }^{5}$ no logró el efecto pretendido en la Junta del Servicio Selectivo de Berkeley, que poco después reclasificó a Foster como "1-A", lo que significa que era apto para el servicio militar. Por eso, en marzo de 1944, mientras el acuerdo de cooperación entre México y Estados Unidos seguía en el aire, Foster tuvo que reportarse a su junta de servicio al otro lado del país en Berkeley. Ocurre, sin em-

${ }^{5}$ En el National Anthropological Archives en Washington, D.C., encontré un documento posterior sin autor ni fecha (ISA, George M. Foster, correspondencia, 1942-1945) que lleva una anotación escrita a mano: "Reescrito por H. W. Dorsey, 1/5/44". Aunque este documento no contiene información respecto de su origen o propósito, y no se sabe si fue enviado a alguien en el ISA o si tiene alguna asociación con ese instituto, su contenido no deja dudas de que la misión del ISA en México se relacionaba directamente con la política exterior de Estados Unidos: "El Dr. Foster irá a México a encargarse de un grupo de científicos norteamericanos que representan el Institute of Social Anthropology de la Smithsonian Institution, quienes promoverán la cooperación y el entendimiento interamericanos mediante su trabajo en las ciencias sociales, un campo en que se busca reemplazar el pensamiento totalitario europeo con el abordaje libre de la democrática ciencia norteamericana. Cooperará con el personal del Instituto Nacional de Antropología e Historia de México en la enseñanza de estudiantes mexicanos a través de cursos universitarios y estudios de campo. A su publicación, los resultados de estos últimos proporcionarán información de fondo importante para el uso de programas de acción que afectan las poblaciones que constituyen a México (énfasis mío). 
bargo, que la primavera en Berkeley se caracteriza por altos índices de polen en el aire, así que al presentarse por su examen físico Foster, quien padecía alergias, lo reprobó y fue clasificado “4-F”, es decir, "no apto para el servicio militar". Gracias a este resultado, estaba libre de ir a México tan pronto como se hicieran los preparativos. Quizá fue "un milagro" que Foster fuera declarado "4-F", pero lo cierto es que este resultado cambió el curso de la antropología norteamericana y mexicana en la siguiente generación.

Finalmente, en junio de 1944, Foster y el geógrafo Donald Brand (elegido ${ }^{6}$ como el geógrafo del IsA para enseñar en la ENAH y hacer trabajo de campo en el pueblo de Quiroga, en la orilla nororiental del lago de Pátzcuaro, Michoacán [Brand 1951]), lograron partir para la ciudad de México. Tras instalarse en una casa en la colonia Lomas de Chapultepec con todo y esposa, hijos y muebles, Foster estaba listo para iniciar su actividad docente y arrancar el programa de investigación. Antes del inicio del semestre otoño-invierno en la ENAH, Foster, Brand y Pablo Velásquez (un tarasco que conocía Estados Unidos y hablaba inglés "bastante bien") viajaron a Michoacán a buscar sitios donde sus alumnos de antropología pudieran tener su primera experiencia de trabajo de campo. La primera idea de Foster era instalarse en Ihuatzio, una comunidad tarasca al oriente del lago de Pátzcuaro, pero los tres investigadores primero llevaron a cabo un sondeo en toda la meseta a fin de confirmar esa elección.

Y así fue que, al finalizar el semestre en diciembre de 1944, Foster, Brand y seis estudiantes (Gabriel Ospina de Colombia, Pablo Velásquez de Charapan, Remy Bastien de Haiti y otros tres mexicanos: Angélica Castro, Chita de la Calle y José Corona Núñez) salieron rumbo a Ihuatzio. Aunque Foster llevaba cartas de presentación oficiales del gobernador del estado y otros funcionarios, el cura local sospechaba que él y su grupo eran misioneros, razón por la cual se opuso a sus actividades e hizo imposible su permanencia allí. Entonces, tres días más tarde Foster decidió que él, Ospina y Velásquez irían a Tzintzuntzan. Por su parte, Brand optó por ir con Corona

\footnotetext{
${ }^{6}$ Resultó que Brand fue elegido cuando el geógrafo Henry Bruman no estuvo disponible por problemas con su clasificación para el servicio militar.
} 
Núñez a la comunidad de Quiroga. Los primeros trabajaron en Tzintzuntzan por dos meses antes de regresar a sus clases del semestre primavera-verano en la ENAH. Foster, Ospina y Velásquez permanecieron en Tzintzuntzan otros cuatro o cinco meses. En un momento hubo dificultades allí que obligaron a Foster a llamar a Borbolla y pedir su ayuda para arreglar el asunto. Borbolla ya conocía Tzintzuntzan porque había participado en periodos de trabajo arqueológico en las ruinas prehispánicas (yácatas) cercanas a ese pueblo. Al llegar, reunió al presidente municipal y su cabildo para explicarles el proyecto. Según comenta Foster en su autobiografía oral, "Cuando quedó claro que estábamos allí por un propósito, y con el apoyo del gobierno, no tuvimos más problemas” (2000b, 135).

Foster logró hacer su análisis y redactar una extensa monografía en sólo un año y luego envió el manuscrito a Steward para su aprobación. La versión final de ese trabajo, Empire’s Children: The People of Tzintzuntzan (versión en español: Hijos del imperio), apareció como Publicación Isa núm. 6 (Foster 1948), pero fue hasta el año 2000 que, gracias a El Colegio de Michoacán, finalmente apareció la versión en español de esta obra (2000a). ${ }^{67}$

En 1946, ya terminada la guerra, Steward llegó a México para visitar el programa del IsA y evaluar sitios donde él mismo pudiera hacer trabajo de campo. En ese momento, le dijo a Foster que iba a salir de Washington para aceptar un puesto de profesor en la Universidad de Columbia, y le pidió aceptar el puesto del director del ISA. Foster aceptó, y poco después él y su familia estaban de viaje a Washington, donde al finalizar el verano ya estaban instalados. Tras la salida de Foster, la Dra. Isabel Kelly (Doctorado de la Universidad de California en Berkeley, 1932) fue nombrada la nueva directora del programa ISA en México. En los treinta, los intereses de Kelly habían cambiado del área de la Gran Cuenca (Great Basin) y California al noroeste de México. Ya había hecho sus primeros trabajos arqueológicos profesionales en Sinaloa, Nayarit y Michoacán, pero

${ }^{7}$ Los antropólogos que hacen investigación a largo plazo en Tzintzuntzan distribuyeron gratis un ejemplar de Los hijos del imperio a cada hogar del pueblo y a muchas familias emigrantes en México y Estados Unidos. 
con la partida de Foster, decidió trasladar el programa de capacitación en el trabajo de campo del proyecto ISA-ENAH al estado de Veracruz donde, con su asistente Ángel Palerm (un español), realizó extensas investigaciones entre la población totonaca de Tajín; los resultados parciales de éstas se encuentran en Publicación IsA núm. 13, The Tajin Totonac (Part 1): History, Subsistence, Shelter and Technology (Kelly y Palerm 1952). Cuando el programa del ISA finalizó, Kelly se dedicó a proyectos de antropología aplicada en México y otros países, además de continuar su análisis de materiales arqueológicos recolectados en años anteriores (Fowler y Kemper 2008).

Foster continuó como director del Instituto (salvo por un lapso en 1949-1950 cuando el Dr. Gordon Willey ocupó el puesto mientras Foster aprovechó una beca de la Fundación Guggenheim para hacer trabajo de campo en Espańa) hasta 1951-1952, año en que el gobierno federal retiró su apoyo al programa. De Washington, regresó a Berkeley, donde llegó a ser integrante del Departamento de Antropología hasta retirarse en 1979.

La siguiente visita de Foster a Tzintzuntzan fue en 1958, cuando le fue otorgada una generosa beca de la Fundación Nacional de Ciencia (National Science Foundation) para estudiar el cambio cultural en Michoacán. A partir de esa fecha siguió haciendo trabajo de campo en Tzintzuntzan ańo con ańo hasta rebasar los 80 ańos de edad. Su libro, Los hijos del imperio (2000a, 401-422), contiene un detallado recuento de su investigación a largo plazo en Tzintzuntzan y de las implicaciones de su trabajo para los métodos y la teoría antropológicos.

Implicaciones de los Proyectos Tarascos para la ANTROPOLOGÍA, ENTONCES Y AHORA

Parece evidente, por este breve examen del trabajo antropológico en la región tarasca -desde Sáenz en Carapan, Swadesh et al. en la región de Paracho, Beals et al. en el área de Cherán y hasta Foster et al. en Tzintzuntzan- que ninguno de estos proyectos se habría realizado sin el apoyo y financiamiento proporcionados por agencias de los gobiernos de México y Estados Unidos. Pocos son los antropólogos que tienen el tiempo y los recursos necesarios para realizar extensos 
programas de trabajo de campo, y mucho menos para sostenerse a sí mismos (y a sus familias) en el campo durante periodos extendidos. Además, sin credenciales y cartas de introducción oficiales expedidas por funcionarios del gobierno resulta muy difícil convencer a la gente local a colaborar en una investigación de campo. Para el antropólogo extranjero el simple acto de entrar en México implica procedimientos y trámites, aunque es suficiente obtener una visa de turista para trabajar en el campo hasta seis meses.

Otro aspecto de estos proyectos es que los antropólogos, lingüistas y otros académicos involucrados estaban limitados por sus responsabilidades laborales lejos de los sitios de sus estudios. Los profesores siempre se veían obligados a volver a sus labores docentes en lugar de permanecer en el campo por tiempo indefinido. El interés en lograr el puesto de titular (tenure) y lograr ascensos y aumentos de sueldo cada año obliga a los antropólogos académicos a publicar los resultados de sus trabajos en foros que sus universidades consideran apropiados. El ejemplo más obvio es la preferencia por publicar artículos en inglés y en las llamadas revistas de "alto nivel", y de entregar monografías a prestigiadas casas editoriales universitarias. Dado este ámbito, no sorprende que pocos resultados de las investigaciones realizadas bajo los auspicios de los proyectos tarascos fueran publicados en revistas en español. Rara vez tenía algún antropólogo recursos económicos propios (la norteamericana Elsie Clews Parsons fue la excepción más notable), o acceso al apoyo institucional a largo plazo (como Sol Tax con la Institución Carnegie en Washington que financió su programa de investigación en Mesoamérica con Robert Redfield entre 1934 y 1944), suficientes para mantenerse independientes del gobierno.

En resumen, está claro que la investigación realizada en $\mathrm{Mi}$ choacán por los diferentes Proyectos Tarascos no fue una empresa sólo de índole intelectual y académica. Esos trabajos se realizaron gracias al sustancial apoyo económico y logístico proveído por numerosas agencias gubernamentales, tanto en México como en Estados Unidos. Este hallazgo me lleva a una reconsideración de la sugerencia que Guillermo de la Peña $(1996,44)$ ofreció en su análisis de "Nacionales y extranjeros en la historia de la antropología mexicana": 
Sugiero que, para los propósitos de este artículo, el concepto de intermediario intelectual (inspirado por el cultural broker de Wolf [1956]) puede resultar de utilidad. Se refiere al papel de un individuo que vuelve inteligibles y aceptables para una comunidad intelectual las ideas científicas y métodos de pesquisa de una comunidad intelectual extranjera. No se trata de una mera labor de traducción, sino de una práctica innovadora; a su vez, la innovación no es simplemente imitativa de lo ajeno: logra una síntesis nueva. Paradójicamente, llega a ser vista como una confirmación de la tradición intelectual propia, y por ello trasciende la moda efímera o la influencia de una personalidad carismática. Incluso puede afirmarse que, en los países "periféricos", el desarrollo científico exitoso depende de la presencia y reproducción de intermediarios intelectuales.

Aquí, De la Peña ofrece una idea práctica que nos ayuda a entender mejor los papeles que los antropólogos, lingüistas y otros académicos jugaron en los Proyectos Tarascos de las décadas de 1930 y 1940. Sin duda, hubo la intención de introducir las corrientes intelectuales norteamericanas en la enseñanza e investigación de la antropología social en México a través de los trabajos que serían realizados en las tierras altas de Michoacán. Pero estaba en juego mucho más que sólo un papel de "intermediarios intelectuales". Sáenz, Swadesh, Beals, Foster y todos sus colaboradores trabajaron con celo "misionero" en inculcar valores que el mundo en guerra ponía en jaque. Como hemos visto, su respuesta consistió en trabajar para el Estado en iniciativas encaminadas a estudiar y transformar un mundo vulnerable a las visiones totalitarias mediante la promulgación de los valores "democráticos" en representación de los gobiernos de México y Estados Unidos. En este sentido, los antropólogos que participaron en los Proyectos Tarascos no fueron simples "intermediarios intelectuales", sino "intermediarios institucionales" en su papel de agentes de sus respectivos gobiernos.

En efecto, los Proyectos Tarascos se hicieron realidad como consecuencia de la convergencia de intereses tanto gubernamentales como antropológicos, y tanto nacionales como extranjeros, en las décadas de 1930 y 1940. Como ha afirmado Ralph Beals (1993, 26), uno de los antropólogos que participó en esos proyectos: "[L]a 
antropología ayudó a la solución de problemas mexicanos y a la vida intelectual del país [...] [L]a antropología mexicana ha tenido un seńalado desarrollo y se ha establecido como un importante campo de investigación, que tiene gran relevancia para la vida y el pensamiento de este país”.

Los Proyectos Tarascos permitieron que la antropología fuera desarrollada por el Estado a un nivel más alto que antes. Como consecuencia, los horizontes de la antropología se ampliaron: su enfoque original en los pueblos indígenas se extendió para incluir a las comunidades campesinas, sus emigrantes y sus lugares de destino en México e, incluso, en Estados Unidos. ${ }^{8} \mathrm{Al}$ mismo tiempo, las perspectivas antropológicas llegaron a tener un papel más importante en la planeación y en los programas que realizaba el Estado. A final de cuentas, el mayor legado del Proyecto Tarasco9 quizá sea esta transformación de la relación entre "Estado y antropología".

\section{REFERENCIAS}

Beals, Ralph L., Cherán: Un pueblo de la sierra tarasca, Zamora, El Colegio de Michoacán, 1992 [original en inglés, 1946]. , "La antropología en el México contemporáneo", en Dos

${ }^{8}$ Esta expansión de los horizontes antropológicos queda manifiesta en el reporte titulado "Research needs in the field of modern Latin American culture", que publicó en 1948 el Committee on Latin American Anthropology del National Research Council. Los integrantes de este comité fueron: Wendell C. Bennett, Ralph L. Beals, John Cooper, Paul Fejos, George M. Foster, Weston LaBarre, Julian Steward, Sol Tax, Charles Wagley, Robert Wauchope y John Gillen (coordinador); antropólogos que representaban a muchas de las instituciones gubernamentales y fundaciones privadas que se mencionan en este artículo.

${ }^{9}$ Después de haber terminado la versión preliminar de este trabajo tuve la suerte de encontrar en internet un documento "Los Proyectos Tarascos: implicaciones actuales", por el etnólogo Carlos García Mora. Este documento me parece muy importante y lo recomiendo al lector (http://www.tsimarhu.com). Según García Mora, su documento es una versión revisada y ampliada del comentario sobre la tesis de historia El Proyecto Tarasco: alfabetización indígena y politica del lenguaje en la Meseta P'urhépecha, 1939-1960 de Tania Ávalos Placencia, la cual fue presentada durante una reunión (31 de marzo del 2007) del Grupo Kw’anískuyarhani de Estudiosos del Pueblo Purépecha. He tenido el gusto de comunicarme con la autora de la citada tesis, y es de esperarse que sería posible intercambiar ideas sobre el tema. 
lecturas de la antropología mexicana, Guadalajara, Universidad de Guadalajara, 1993, 5-29 [original en inglés, 1976].

Beals, Ralph L. y Evelyn Hatcher, "Diet of a Tarascan Village", América Indígena, vol. 3, 295-304, 1943.

Beals, Ralph L. y Pedro Carrasco, "Games of the Tarascan Indians", American Anthropologist, vol. 46, 516-522, 1944.

Beals, Ralph L., Pedro Carrasco y Thomas McCorkle, House and House Use of the Sierra Tarascans, Washington, Smithsonian Institution, Institute of Social Anthropology, Publication núm. $1,1944$.

Bennett, Wendell C., The Ethnogeographic Board, Washington, Smithsonian Institution, Miscellaneous Collections, vol. 107, núm. 1, 1947.

Brand, Donald D., Quiroga: A Mexican Municipio, Washington, Smithsonian Institution, Institute of Social Anthropology, Publication núm. 11, 1951.

Cámara Barbachano, Fernando, "Algunos antecedentes del origen y desarrollo de la Escuela Nacional de Antropología e Historia”, en Manuel Gamio y Raúl Noriega, comps., A William Cameron Townsend en el XXV aniversario del I.L.V., 1961, 33-41. Colegio de Etnólogos y Antropólogos Sociales, A.C., El I.L.V. en México: Dominación ideológica y ciencia social, declaración José C. Mariategui, México, Nueva Lectura, 1979.

Committee on Latin American Anthropology of the National Research Council, "Research needs in the field of modern Latin American culture”, Acta Americana, vol. 6, núms. 1-2, 1948, $17-25$.

Cramer, Gisela y Ursula Prutsch, "Nelson A. Rockefeller's Office of Inter-American Affairs (1940-1946) and Record Group 229", Hispanic American Historical Review, vol. 86, núm. 4, 2006, 785-806.

De la Peña, Guillermo, "Nacionales y extranjeros en la historia de la antropología mexicana", en Mechthild Rutsch, comp., La historia de la antropología en México: fuentes y transmisión, México, Seminario de Historia, Filosofía y Sociología de la Antropología Mexicana, Universidad Iberoamericana, Instituto Nacional Indigenista y Plaza y Valdés, 1996, 41-81. 
Del Castillo, Ignacio M., "La alfabetización en lenguas indígenas: el Proyecto Tarasco", América Indígena, vol. 5, núm. 2, abril, 1945, 139-151.

Foster, George M., “The Institute of Social Anthropology”, en Walter Goldschmidt, ed., The Uses of Anthropology, Washington, American Anthropological Association, Special Publication, núm. 11, 1979, 205-216.

Los hijos del imperio: la gente de Tzintzuntzan, Zamora, El Colegio de Michoacán, 2000a [original en inglés, 1948].

, An Anthropologist's Life in the Twentieth Century: Theory and Practice at UC Berkeley, the Smithsonian, in Mexico, and with the World Health Organization, una historia oral realizada en 1998 y 1999 por Suzanne B. Reiss, Berkeley, Regional Oral History Office, The Bancroft Library, 2000b.

Fowler, Catherine S. y Robert V. Kemper, "Una vida en el campo: Isabel T. Kelly, 1906-1982”, en Isabel T. Kelly, Excavaciones en Chametla, Sinaloa, México, Siglo XXI, El Colegio de Sinaloa e INAH, 2008, IX-XLIII.

Gamio, Manuel y Raúl Noriega, comps., A William Cameron Townsend en el vigesimoquinto aniversario del Instituto Lingüistico de Verano, México, 1961.

Ginzberg, Eitan, Lázaro Cárdenas. Gobernador de Michoacán (1928-1932), Zamora, El Colegio de Michoacán, Universidad Michoacana de San Nicolás de Hidalgo, 1999.

Hewitt de Alcántara, Cynthia, Anthropological Perspectives on Rural Mexico, Londres, Routledge y Kegan Paul, 1984.

Jiménez Moreno, Wigberto, "Origen y desarrollo de la Escuela Nacional de Antropología e Historia”, Revista Mexicana de Estudios Antropológicos, vol. 10, 1948-1949, 135-142.

Kelly, Isabel y Ángel Palerm, The Tajin Totonac (Part 1): History, Subsistence, Shelter and Technology, Publicación núm. 13, Washington, Smithsonian Institution, Institute of Social Anthropology, 1952.

Kemper, Robert V., "Del nacionalismo a la internacionalización: el desarrollo de la antropología mexicana, 1934-1946”, en Dos lecturas de la antropología mexicana, Guadalajara, Universidad de 
Guadalajara, 1993, 31-61 [original en inglés, 1984].

Kirchhoff, Paul, "Mesoamérica”, Acta Americana, vol. 1, núm. 1, enero-marzo, 1943, 92-107.

LAMEIRAS, José, "La antropología en México: panorama de su desarrollo en lo que va del siglo", Ciencias sociales en México: Desarrollo y perspectiva, México, El Colegio de México, 1979, 107-180.

Rubín de la Borbolla, Daniel F. y Ralph L. Beals, “The Tarasca Project: A cooperative enterprise of the National Polytechnic Institute, Mexican Bureau of Indian Affairs, and the University of California", American Anthropologist, vol. 42, 1940, 708-712.

RuTsCH, Mechthild, comp., La historia de la antropología en México: fuentes y transmisión, México, D.F., Seminario de Historia, Filosofía y Sociología de la Antropología Mexicana, Universidad Iberoamericana, Instituto Nacional Indigenista y Plaza y Valdés, 1996.

SÁEnz, Moisés, Carapan: bosquejo de una experiencia, Lima, 1936 [publicado como Carapan, por el Departamento de Promoción Cultural del Gobierno de Michoacán, Morelia, 1970].

Swadesh, Morris, Wigberto Jiménez Moreno, Juan Luna CárDENAs, Norman McQuown, Jorge Vivó y Daniel Rubín DE LA Borbolla, Memoria de la Primera Asamblea de Filólogos y Lingüistas, México, 1939.

Wolf, Eric R., "Aspects of group relations in a complex society", American Anthropologist, vol. 58, 1956, 1065-78. 